\title{
A PLEA FOR THE FORGOTTEN FACTOR IN CHEMICAL TRAINING.
}

Mr. W. R. Cooper, M.A., B.Sc., read a Paper entitled "A Plea for the Forgotten Factor in Chemical Training."

In considering the training of the chemical engineer nine people out of ten (and even more) take the view that so long as the student at the end of his course has a good knowledge of theoretical chemistry and laboratory methods, and has some notion of the main manufacturing processes, as disclosed by textbooks, his training is complete. If he has passed through one of the few laboratories where commercial processes entering into manufacture may be studied, so much the better.

It is, of course, true that no manufacturing concern can be thoroughly and permanently successful unless its technical processes are fully understood. The technical aspect is therefore always emphasized. Yet, however perfectly the theoretical and technical knowledge of the young chemist may be developed during his technical training, there is usually an all-important factor which is wholly neglected, namely $€ \mathrm{~s}$. d. In other words, will any particular process pay? Of course this question is very unscientific, and I feel I must almost apologize for bringing it before a scientific society ; but the fact remains that the end and aim of every commercial chemical process is to make a profit or to reduce a loss (which is really the same thing), and yet this all-important point of view is omitted from the student's course.

If cost could be disregarded the power of the scientific man would be much increased. Unfortunately, results obtained regardless of cost are of no commercial value. In days to come it may weil be that one of our chemists will discover the transmutation of lead into gold, and the daily Press will forthwith assure their readers that, as soon as this memorable discovery can be put to work, gold will be comparatively worthless, because a hundredweight of lead costs much less than an ounce of gold. But in all probability Nature has so adjusted the elements that an ounce of transmuted lead will cost a good deal more than an ounce of gold as obtained by present-day methods, when all the charges are taken into account. The inclusion of all the charges is where the trouble comes in ; and it is in this way that a competitive process frequently turns out to be much less formidable than was anticipated by its promoters.

The idea of introducing the factor of cost is often distasteful to the teacher, probably in proportion to his academic attainments; but to imagine that a commercial problem is on a lower plane than one that is purely scientific is a mistake, for it includes the additional factor of cost, which makes it all the more difficult of solution. The methods used may be less refined and less accurate, but that is only a matter of degree. Moreover, the probability is that the working on a commercial scale introduces complications which in 
the laboratory were not apparent, and thus there are fresh fields of difficulty unforeseen by the academic worker.

A few points may be mentioned by way of illustration.

A fundamental characteristic of any stable commercial enterprise is that it should be run at a profit. This is a truism, and yet, as a rule, the young chemical engineer has the haziest notion of what is meant by profit. He generally thinks that so long as the price obtained exceeds the cost of material and labour, together with a small margin towards rent and management, a profit is being made. This, of course, is far from the case.

It seems desirable that there should be some appreciation of the fact that manufacturing cost, even when correctly determined, is only one part of the total. The cost of selling the product is equally important and in some cases is much greater than the cost of manufacture. In the first few years of an enterprise this may be particularly important.

Then there is the question of the depreciation of plant, which may be very heavy under certain conditions, and the kindred problem of obsolescence. The latter may be forced upon the user through the medium of competition, it being found that an old type of plant must be discarded in favour of a newer type if the costs are to be reduced to meet competition.

Further, there is the division of costs into "overhead" and running costs, the former being the costs which are incurred simply by being ready to manufacture and to sell, apart from the cost of actual production. The overhead cost as a total per annum is fixed for the time being, and thus the extreme importance of a maximum output for a given overhead cost per annum is seen if the cost of the product is to be a minimum. This means that the full utilization of plant (i.e. of capital) is vital and may necessitate the employment of more than one shift.

With this part of the subject is bound up the question of the utility factor or commercial efficiency of plant as distinct from technical efficiency. The technical efficiency of a plant when at work may be quite high; in other words, the waste of materials or of energy in obtaining the product may be small. Yet, if it is necessary to interrupt the working of plant for discharging, charging, adjustment, etc., the utility factor (i.e. the ratio of the quantity of material actually produced during, say, a year to the quantity that would be produced if the plant were working continuously under the best conditions) may be low. It may be worth while to have a lower technical efficiency if this increases the utility factor, and it is on these lines that the merits of continuous versus discontinuous processes must be weighed.

An equally important point in many cases, but one which may not be appreciated by the young chemist, is the speed at which a reaction will take place. Take, for instance, the case of a catalytic process. If the speed of reaction can be doubled, this may make all the difference between success and failure commercially, owing to the difference in the capital required, or it may enable the manufacturer who secures this end by investigation to compete more readily with other manufacturers.

The above points will serve as a few examples to illustrate what I mean by the "forgotten factor." By some it is urged that such matters should receive no attention in our educational courses; that the would-be chemical engineer should study merely chemistry, theoretical and applied. The view is taken that if the student is well grounded in this way he will soon pick up the rest when he enters the works, assuming that the need ever arises for such knowledge. In the case of large works this may be true enough ; the young chemist may possibly not come across the commercial side at all, and when he does so he will find himself among those who already have sound 
views on commercial efficiency. But in small works and in other lines of activity this is often not the case ; the young chemist may find himself without such guidance, and may nevertheless be called upon to decide or advise upon processes on broad commercial lines. It therefore seems a mistake to leave the profit side of chemical operations entirely untouched. I feel that if this aspect were impressed upon the young chemist we should not so often see the spectacle of unsound chemical processes being put forward by the chemist and even backed by men of the highest repute in the world of chemistry. They would realize that there is much to be considered beyond the fundamental chemical reactions of a given process.

Something, though not much, is being done by our modern universities to deal with commercial matters. For example, Birmingham University has established a Faculty of Commerce. One of the two alternatives presented by the syllabus is for the benefit of students who expect to be engaged in the commercial conduct of manufacturing businesses, and such students are permitted to devote about one-third of their time to the study of applied science. For a commercial training this division of time would, no doubt, be satisfactory, but would, of course, be quite in the wrong proportion for the chemical engineer. Actually, as far as I am aware, nothing is dorie by our schools at present to meet the case here considered.

It is generally recognized that any serious prolongation of, say, a four years' course is undesirable. It appears to me, however, that this would not be necessary to secure the desired end. What is required is a grounding in sound principles rather than an acquaintance with much detail, and probably this could be accomplished without difficulty in a course of three months at the conclusion of the technical course. Undoubtedly there are many other subjects of which some knowledge is desirable, such as works organization, methods of remuneration, economics and the law of contracts, but these can be left more readily for the student to consider when they are forced upon him.

In conclusion, let me say that while I do not suggest any very drastic alteration in our methods, I do put forward a strong plea for the consideration of the one factor which is generally omitted from every college training, but which nevertheless regulates our attitude towards every process in commercial working. 